\title{
An unconditionally stable time-domain discretization on cartesian meshes for the simulation of nonuniform magnetized cold plasma
}

\author{
Wouter Tierens ${ }^{\mathrm{a}, *}$, Daniël De Zutter ${ }^{\mathrm{a}}$ \\ ${ }^{a}$ Ghent University, Department of Information Technology, Sint-Pietersnieuwstraat 41, \\ Ghent, Belgium
}

\begin{abstract}
In this paper an unconditionally stable, spatially and temporally implicit time-domain discretization for nonuniform magnetized cold plasma is developed. The discrete dispersion relation is free of spurious solutions and approximates the continuous dispersion relation for well-resolved wavelengths and frequencies $\left(k \Delta \ll \pi, \omega \Delta_{t} \ll \pi\right)$. For a specific choice of parameters, the discrete dispersion relation approximates the continuous dispersion relation for all wavelengths and frequencies up to the Nyquist limit. A few examples, amongst them one involving mode conversion, illustrate the new method.
\end{abstract}

Keywords: Cold plasma, time domain

\section{Introduction}

A lot of research has been performed into the time-domain simulation of electrodynamics in non-trivial media $[1,2,3,4]$, including Lorentz dielectrics [1], lasing media [5], unmagnetized plasmas [6], and magnetized plasmas [7]. Modelling such media is usually done using an extra differential equation (ADE, auxiliary differential equation) in addition to the classical Maxwell's equations. In magnetized plasmas, this approach is especially difficult because of the nature of the ADE, which is naturally discretized on a collocated grid, while Maxwell's equations are usually discretized on staggered

\footnotetext{
*Corresponding author

Email addresses: Wouter.Tierens@Ugent.be (Wouter Tierens), Daniel.Dezutter@Ugent.be (Daniël De Zutter)
} 
grids. Explicit solutions have been proposed [7] and are stable provided the vacuum Courant condition is obeyed. This is problematic when simulating phenomena whose wavelength is much shorter than the vacuum wavelength $\lambda_{v a c}=2 \pi c / \omega$, like the waves that occur in mode conversion layers $[7,8,9,10]$. In that case, to resolve the phenomena in space (i.e. the spatial discretization length is a reasonable fraction of the wavelength) a very small time step must be chosen, where the time step is a very small fraction of the period of the sinusoidal source. In this paper, we present a fully implicit time-domain approach which is unconditionally stable such that $\Delta$, the space step, and $\Delta_{t}$, the time step, can be chosen independently.

Section 2 of this paper presents the cold magnetized plasma equations. The new spatial and temporal implicit discretization we propose is explained in Section 3.1 together with a detailed discussion of the time-stepping (Section 3.2) and a proof of its stability for periodic boundary conditions (Section 3.3). A more general proof is as yet elusive but the numerical examples confirm stability in other cases as well. Finally Section 3.4 discusses the discretized version of the dispersion relation and the choice of the time step $\Delta_{t}$ versus the space step $\Delta$. In Section 4 special attention is devoted to the efficient solution of the set of equations that has to be solved at each time step. Numerical examples are presented in Section 5 followed by the conclusions in Section 6.

\section{Basic equations}

Smithe [7] proposes the following equations to describe the time-domain behavior of cold magnetized plasmas

$$
\begin{aligned}
-\frac{\partial \vec{B}}{\partial t} & =\vec{\nabla} \times \vec{E} \\
\epsilon_{0} \frac{\partial \vec{E}}{\partial t} & =-\sum_{s} \vec{J}_{s}+1 / \mu_{0} \vec{\nabla} \times \vec{B} \\
\frac{\partial \overrightarrow{J_{s}}}{\partial t} & =\epsilon_{0} \omega_{s}^{2} \vec{E}-\vec{\Omega}_{s} \times \vec{J}_{s}
\end{aligned}
$$

where $\vec{J}_{s}$ is the current of the sth particle species (electron current, proton

current, ...), $\omega_{s}=\sqrt{\frac{n_{s} q_{s}^{2}}{m_{s} \epsilon_{0}}}$ is the corresponding plasma frequency (where $n_{s}$ is the particle density, $q_{s}$ is the charge and $m_{s}$ the mass) and $\overrightarrow{\Omega_{s}}=q_{s} \overrightarrow{B_{0}} / m_{s}$ is 
the cyclotron frequency due to the background magnetic field $\overrightarrow{B_{0}}$. All of these quantities may be position-dependent, but the background quantities $\overrightarrow{B_{0}}$ and $n_{s}$ are time-independent (i.e. (1)-(3) represent small linearized changes on a constant background).

By rescaling the fields, (1)-(3) can be rewritten in a convenient antisymmetric form

$$
\begin{aligned}
\overrightarrow{\mathcal{B}} & =\frac{1}{\sqrt{\mu_{0}}} \vec{B} \\
\overrightarrow{\mathcal{E}} & =\sqrt{\epsilon_{0}} \vec{E} \\
\overrightarrow{\mathcal{J}} & =\epsilon_{0}^{3 / 2} \omega_{s} \vec{J}_{s} \\
\frac{\partial \overrightarrow{\mathcal{B}}}{\partial t} & =-c \vec{\nabla} \times \overrightarrow{\mathcal{E}} \\
\frac{\partial \overrightarrow{\mathcal{E}}}{\partial t} & =-\sum_{s} \omega_{s} \overrightarrow{\mathcal{J}}_{s}+c \vec{\nabla} \times \overrightarrow{\mathcal{B}} \\
\frac{\partial \overrightarrow{\mathcal{J}}}{\partial t} & =\omega_{s} \overrightarrow{\mathcal{E}}-\vec{\Omega}_{s} \times \overrightarrow{\mathcal{J}}_{s}
\end{aligned}
$$

which is what we will use throughout this paper.

Spurious solution-free discretization methods for Maxwell's equations are well-known [11]. However, in these equations a new difficulty arises: the constitutive equation needs all current and electric field components at the same position. This makes it difficult to combine with staggered approaches such as the Yee cell. Injudiciously chosen interpolation schemes risk introducing non-physical "spurious" modes by introducing a cosine factor in some parts of the discrete dispersion relation.

Smithe [7] solves these problems in an explicit FDTD framework by repeatedly interpolating from Yee cell positions to collocated positions and back.

Here, we will present an implicit time-domain discretization scheme that solves these problems. By virtue of being implicit in time, the time step $\Delta_{t}$ does not have to obey the Courant limit. On the other hand, at every time step a (sparse) set of equations has to be solved, which is a disadvantage relative to explicit discretizations but we will show later in this paper that this sparse system can be solved very efficiently. The proposed discretization is also implicit in space, and has the remarkable property of being able to reproduce the continuous dispersion relation for all resolvable $k$ and $\omega$ and 
not only for sufficiently small $k$ and $\omega$. This is only true fo a specific choice of $\Delta_{t}$, as discussed in the sequel.

\section{Spatial and temporal discretization}

\subsection{Generalities}

Consider e.g. the $y$-component of (7)

$$
\frac{\partial \mathcal{E}_{x}}{\partial z}-\frac{\partial \mathcal{E}_{z}}{\partial x}=-\frac{1}{c} \frac{\partial \mathcal{B}_{y}}{\partial t}
$$

We discretize space-time using $4 \mathrm{D}$ "cubical" cells of side $\Delta_{x}, \Delta_{y}, \Delta_{z}, \Delta_{t}$. All fields are written as linear combinations of products of $1 \mathrm{D}$ rooftop functions centered on cell corners. It is important to emphasize here that all field components are collocated at the cell corners. The basis-function associated with a corner at $\left(x_{0}, y_{0}, z_{0}, t_{0}\right)$ is

$$
f\left(x, y, z, t, x_{0}, y_{0}, z_{0}, t_{0}\right)=r\left(x, x_{0}, \Delta_{x}\right) r\left(y, y_{0}, \Delta_{y}\right) r\left(z, z_{0}, \Delta_{z}\right) r\left(t, t_{0}, \Delta_{t}\right)
$$

where the "rooftop" function $r\left(x, x_{0}, \Delta\right)$ is defined as

$$
r\left(x, x_{0}, \Delta\right)=\left\{\begin{array}{cc}
0 & x<x_{0}-\Delta \\
0 & x>x_{0}+\Delta \\
\frac{x-x_{0}}{\Delta}+1 & x \in\left[x_{0}-\Delta, x_{0}\right] \\
1-\frac{x-x_{0}}{\Delta} & x \in\left[x_{0}, x_{0}+\Delta\right]
\end{array}\right.
$$

To "test" (10), we use the following testing functions

$$
b\left(x, x_{0}^{\prime}+\Delta_{x} / 2, \Delta_{x}\right) r\left(y, y_{0}^{\prime}, \Delta_{y}\right) b\left(z, z_{0}^{\prime}+\Delta_{z} / 2, \Delta_{z}\right) b\left(t, t_{0}^{\prime}+\Delta_{t} / 2, \Delta_{t}\right)
$$

where a "pulse" function for the $x$-direction and centered at $x_{c}$ is given by

$$
b\left(x, x_{c}, \Delta\right)=\left\{\begin{array}{cc}
0 & x<x_{c}-\Delta / 2 \\
0 & x>x_{c}+\Delta / 2 \\
1 & x \in\left[x_{c}-\Delta / 2, x_{c}+\Delta / 2\right]
\end{array}\right.
$$

and of course similarly for $y, z$ and $t$. Note that derivatives of rooftop functions are linear combinations of pulse functions. Further remark that (10) features derivatives with respect to $\mathrm{x}, \mathrm{z}$ and $\mathrm{t}$ and not with respect to $\mathrm{y}$. The testing function (13) is chosen such that one of its factors is a rooftop 
function with respect to this y-coordinate, while for the other coordinates pulse functions are used.

Let us now return to $(10)$. Representing $\mathcal{E}_{x}, \mathcal{E}_{z}$ and $\mathcal{B}_{y}$ by expansions of the form (11), we remark that at a given $y=y_{0}$ coordinate, we can factor out $r\left(y, y_{0}, \Delta_{y}\right)$, allowing to rewrite (10) as

$$
r\left(y, y_{0}, \Delta_{y}\right)\left(\frac{\partial \mathcal{E}_{x}}{\partial z}-\frac{\partial \mathcal{E}_{z}}{\partial x}+\frac{1}{c} \frac{\partial \mathcal{B}_{y}}{\partial t}\right)_{x, z, t}=0
$$

where the subscript indicates the remaining space- and time- dependence of the quantity in brackets $\left(\mathcal{E}_{x}, \mathcal{E}_{z}\right.$ and $\mathcal{B}_{y}$ are no longer identical to their values in (10) as we we have factored out the $y$-dependence, but the notation is easily understood). Testing this with the proper testing function pertaining to the $y$-direction, gives as a weighting factor an integral of the form

$$
\int_{-\infty}^{\infty} r\left(y, y_{0}, \Delta_{y}\right) r\left(y, y_{0}^{\prime}, \Delta_{y}\right) d y \approx \Delta_{y} \delta_{y_{0}, y_{0}^{\prime}}
$$

if the integral is mass lumped. Trapezoidal integration, or "mass lumping" is a convenient technique to make matrices sparser or easier to invert (the impact of mass lumping in our approach will become clear in section 4). It has been extensively used in FDTD and FE (e.g. [12]). The integral in principle extends over the whole space $(-\infty$ to $\infty)$ but the integrands differ from zero only in some finite region. The remaining weighting integral now becomes

$$
\begin{gathered}
\int_{-\infty}^{\infty}\left(\frac{\partial \mathcal{E}_{x}}{\partial z}-\frac{\partial \mathcal{E}_{z}}{\partial x}+\frac{1}{c} \frac{\partial \mathcal{B}_{y}}{\partial t}\right)_{x, z, t} b\left(x, x_{0}^{\prime}+\Delta_{x} / 2, \Delta_{x}\right) \\
\cdot b\left(t, t_{0}^{\prime}+\Delta_{t} / 2, \Delta_{t}\right) b\left(z, z_{0}^{\prime}+\Delta_{z} / 2, \Delta_{z}\right) d x d z d t
\end{gathered}
$$

Here, "pulse-pulse" and "pulse-rooftop" testing integrals do not need to be mass lumped. The integrals can easily be calculated exactly and attempting to mass lump them would sample points just at the edge of a pulse, where its function value is not well-defined. We could easily remedy this by introducing a limiting procedure into the mass lumping process, but it is far easier to simply calculate the perfectly well-defined integrals (and this leads to the same result anyway). Consider the first term of (17)

$$
\begin{aligned}
\int_{-\infty}^{\infty} & \left(\frac{\partial \mathcal{E}_{x}}{\partial z}\right)_{x, z, t} b\left(x, x_{0}^{\prime}+\Delta_{x} / 2, \Delta_{x}\right) \\
& \cdot b\left(t, t_{0}^{\prime}+\Delta_{t} / 2, \Delta_{t}\right) b\left(z, z_{0}^{\prime}+\Delta_{z} / 2, \Delta_{z}\right) d x d z d t
\end{aligned}
$$


where

$$
\begin{aligned}
\left(\frac{\partial \mathcal{E}_{x}}{\partial z}\right)_{x, z, t} & =r\left(x, x_{0}, \Delta_{x}\right) r\left(t, t_{0}, \Delta_{t}\right) \\
& \cdot\left(b\left(z, z_{0}-\Delta_{z} / 2, \Delta_{z}\right)-b\left(z, z_{0}+\Delta_{z} / 2, \Delta_{z}\right)\right) / \Delta_{z}
\end{aligned}
$$

This term becomes

$$
\begin{aligned}
\int_{-\infty}^{\infty} r & \left.r, x_{0}, \Delta_{x}\right) b\left(x, x_{0}^{\prime}+\Delta_{x} / 2, \Delta_{x}\right) d x \\
& \cdot \int_{-\infty}^{\infty} r\left(t, t_{0}, \Delta_{t}\right) b\left(t, t_{0}^{\prime}+\Delta_{t} / 2, \Delta_{t}\right) d t \\
& \cdot \frac{1}{\Delta_{z}}\left(\int_{-\infty}^{\infty} b\left(z, z_{0}-\Delta_{z} / 2, \Delta_{z}\right) b\left(z, z_{0}^{\prime}+\Delta_{z} / 2, \Delta_{z}\right) d z\right. \\
& \left.-\int_{-\infty}^{\infty} b\left(z, z_{0}+\Delta_{z} / 2, \Delta_{z}\right) b\left(z, z_{0}^{\prime}+\Delta_{z} / 2, \Delta_{z}\right) d z\right)
\end{aligned}
$$

The $x$-integration gives either $\frac{\Delta_{x}}{2}$ or 0 (for $x_{0}=x_{0}^{\prime}, x_{0} \neq x_{0}^{\prime}$, resp.), the $t$ integration gives either $\frac{\Delta_{t}}{2}$ or 0 , and the $z$ integration (including the $\frac{1}{\Delta_{z}}$ factor) gives either 1 (if $z_{0}=z_{0}^{\prime}$ ), -1 (if $z_{0}=z_{0}^{\prime}+\Delta_{z}$ ) or 0 (otherwise). Hence the testing integral for $\frac{\partial \mathcal{E}_{x}}{\partial z}$ for a specific $\mathcal{E}_{x}$ basis-function gives 0 if the testing function does not overlap with the $\mathcal{E}_{x}$ basis-function and $\pm \frac{1}{4} \Delta_{y} \Delta_{x} \Delta_{t}$ if the the testing function does overlap with the $\mathcal{E}_{x}$ basis-function. If we divide away the testing function 4 -volume $\Delta_{y} \Delta_{x} \Delta_{t} \Delta_{z}$ we get $\pm \frac{1}{4} \frac{1}{\Delta_{z}}$ corresponding to $\frac{1}{4}$ of the discretized derivative with respect to $z$. There are in total $8 \mathcal{E}_{x}$ basisfunctions that overlap with any given test function ( 4 at $t, 4$ at $t+\Delta_{t}$ ) and the testing procedure averages their $z$-derivative ( 4 such derivatives, each containing an extra factor $\left.\frac{1}{4}\right)$ at the center of a $(x, z, t)$-cube. There are also 8 basis-functions corresponding with each of the other two field components $\left(\mathcal{E}_{z}\right.$ and $\left.\mathcal{B}_{y}\right)$ in $(10)$ and their derivatives too, are interpolated to the cube center. This finally leads to a discretized version of (10) that is implicit both in space and in time.

Above, we have explained how the proposed spatial and temporal discretization works for the y-component of (10). Similarly, for all other components of Maxwell's equations a dedicated testing function similar to (13) must be constructed in such a way that derivatives w.r.t. coordinates (including time) occurring in the continuous equation give rise to the corresponding pulse function factor in the testing function, and coordinates for which no 
derivatives occur in the continuous equation give rise to the corresponding rooftop factor. In particular, in constitutive equation (9), only a time derivative is present and its testing function will be a product of three spatial rooftops and one temporal pulse function. Summarizing, the proposed discretization scheme separately considers each scalar first-order partial differential equation (i.e. one of the components of Maxwell's curl equations or one of the components of the constitutive equation), then constructs a 3D cube (or line in the case of the constitutive equation) whose edges are the directions along which the scalar PDE contains derivatives (including temporal derivatives, the cube is not purely spatial). The derivatives occurring in the PDE are then defined at centers of the edges of this cube. Next, all terms of the scalar PDE are interpolated to the center of the cube. The resulting equation at this point is the discretized end result. Note that the 6 scalar PDE's of Maxwel's curl equations contain derivatives in different directions and hence are discretized at the center of different cubes. For a concrete example in 1D, see Fig. 1. In this figure we again consider (10) which in 1D simplifies to $\frac{\partial \mathcal{E}_{x}}{\partial z}=-\frac{1}{c} \frac{\partial \mathcal{B}_{y}}{\partial t}$. In this case the $(z, t)$ "cube" center is $O$. If $O$ is at $z=n \Delta$ and $t=m \Delta_{t}$, field values in points 3 and 4 are at $t=(m+1 / 2) \Delta_{t}$, field values in points 1 and 2 are at $t=(m-1 / 2) \Delta_{t}$, field values in points 1 and 3 are at $z=(n-1 / 2) \Delta$, while field values in points 2 and 4 are at $z=(n+1 / 2) \Delta$.

As remarked by one of the reviewers, the results of our basis and test function procedure, could, as in Fig. 1, be directly represented in terms of a finite-difference interpolation scheme. We have chosen to introduce the pulse-rooftop approach as this way of discretizing space is very well-known in the electromagnetics community [13] and also because we believe this approach is more easily extendable to other coordinate systems, to higher-order approximations and even to unstructured meshes. As further remarked by another reviewer, researchers from the finite element community might prefer to express our approximation in more rigorous terms [14]. In these terms the basis or trial space consists of a $C_{0}$-continuous $P_{1}$ Lagrange basis in spacetime for all magnetic field, electric field and current components, yielding a non-staggered approximation. The test space is slightly more complicated due to the fact that the curl-operator features no $i$-th spatial derivative for the $i$-th vector component. Consequently, for Maxwell's curl equations the test space consists of a $C_{0}$-continuous $P_{1}$ Lagrange basis in the spatial directions with missing derivative and a $P_{0}$ basis for all other components. For the constitutive equation, which contains no spatial derivatives, the test space 

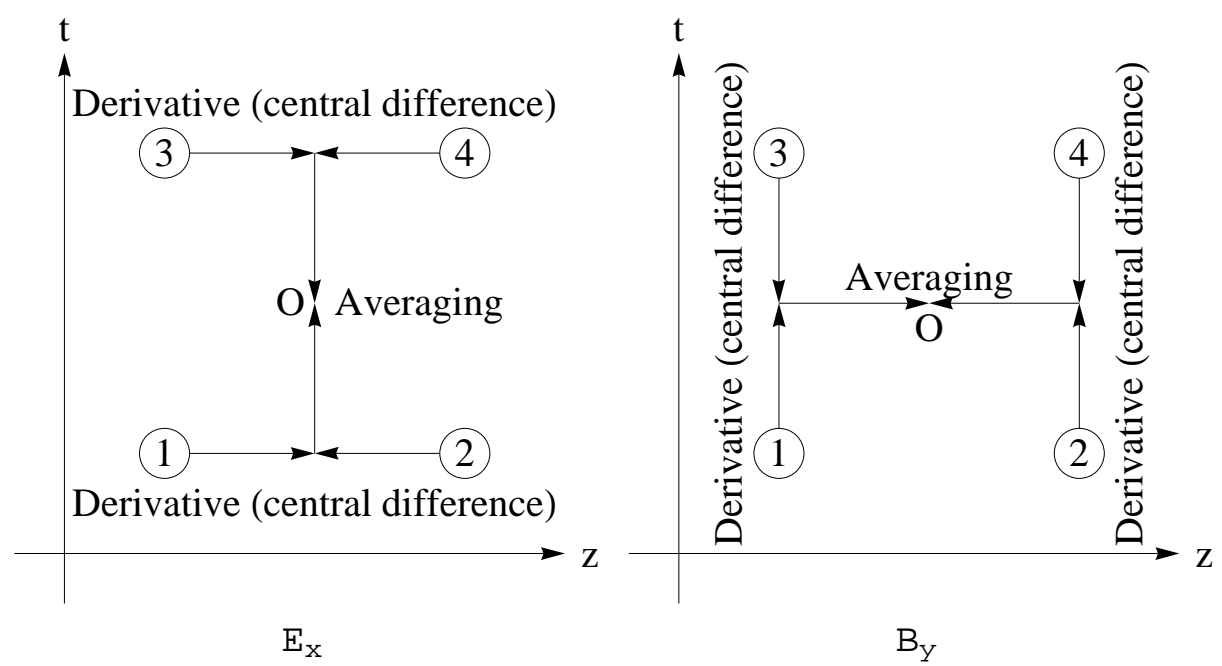

Figure 1: discretization of (10) with only 1 spatial dimension $(z)$. The discretized equation for this cell is $\frac{\left(\mathcal{E}_{x, 2}-\mathcal{E}_{x, 1}\right)+\left(\mathcal{E}_{x, 4}-\mathcal{E}_{x, 3}\right)}{2 \Delta}=\frac{-1}{c} \frac{\left(\mathcal{B}_{y, 3}-\mathcal{B}_{y, 1}\right)+\left(\mathcal{B}_{y, 4}-\mathcal{B}_{y, 2}\right)}{2 \Delta_{t}}$.

consists of a $C_{0}$-continuous $P_{1}$ Lagrange basis in all spatial directions and a $P_{0}$ basis in time.

The reader might wonder why we opted for the above discretization scheme. Let us return to (10). After inserting a complex exponential wave solution $\exp \left(j k_{x} x\right) \exp \left(j k_{y} y\right) \exp \left(j k_{z} z\right) \exp (j \omega t)$ for each of the field components (as customary when investigating the dispersive behavior of the discretized equations), taking the discrete derivative and interpolating to the center of the $(x, z, t)$-cube, the 3 terms turn out to be proportional to:

$\frac{\partial \mathcal{E}_{x}}{\partial z} \propto \sin \left(k_{z} \frac{\Delta_{z}}{2}\right) \cos \left(k_{x} \frac{\Delta_{x}}{2}\right) \cos \left(\omega \frac{\Delta_{t}}{2}\right) \exp \left(i k_{x} x\right) \exp \left(i k_{y} y\right) \exp \left(i k_{z} z\right) \exp (i \omega t)$

$\frac{\partial \mathcal{E}_{z}}{\partial x} \propto \sin \left(k_{x} \frac{\Delta_{x}}{2}\right) \cos \left(k_{z} \frac{\Delta_{z}}{2}\right) \cos \left(\omega \frac{\Delta_{t}}{2}\right) \exp \left(i k_{x} x\right) \exp \left(i k_{y} y\right) \exp \left(i k_{z} z\right) \exp (i \omega t)$

$\frac{\partial \mathcal{B}_{y}}{\partial t} \propto \sin \left(\omega \frac{\Delta_{t}}{2}\right) \cos \left(k_{x} \frac{\Delta_{x}}{2}\right) \cos \left(k_{y} \frac{\Delta_{y}}{2}\right) \exp \left(i k_{x} x\right) \exp \left(i k_{y} y\right) \exp \left(i k_{z} z\right) \exp (i \omega t)$

After dividing each term of the discretized equation (10), i.e. (21)-(23), by a factor $\cos \left(k_{x} \frac{\Delta_{x}}{2}\right) \cos \left(k_{y} \frac{\Delta_{y}}{2}\right) \cos \left(\omega \frac{\Delta_{t}}{2}\right)$, the only difference with the continuous 
counterpart for a complex exponential wave solution is that all $k_{i}(i=x, y, z)$ and $\omega$ are replaced by $\tan \left(k_{i} \Delta_{i} / 2\right) 2 / \Delta_{i}$ or $\tan \left(\omega \Delta_{t} / 2\right) 2 / \Delta_{t}$. This is precisely why we used such unorthodox basis- and testing-functions : $k_{i}$ and $\omega$ are replaced by tangent-functions, correctly approximating the continuous behavior for low values of $k_{i}$ and $\omega$. This is the only change to the dispersion relation. There are no further artifacts in the discretization scheme: purely local interactions remain purely local. In contrast, testing (1)-(3) with classical staggered grid basis-functions (such as the ones used in [12]) leads to factors of the form $\cos \left(k_{i} \frac{\Delta_{i}}{2}\right)$ in some terms but not in others, which may cause spurious solutions at high wave numbers. It is also clear that there are no spurious roots in the discrete dispersion relation, as will also become clear from the examples in Section 4, despite warnings against using basisfunctions of the same order in all directions, e.g. in chapter 5 of [15]. We have circumvented these problems by instead using testing functions of different order in different directions.

\subsection{Time-stepping}

We have proposed a discretization scheme that is both implicit in space and time and will now study in more detail how the time-stepping is performed by turning to the set of equations that has to be solved at every time step. To this end all field component are gathered in a vector $V=\left[\mathcal{B E}_{\mathcal{J}}\right]^{T}$. When applying our discretization procedure to the spatial derivatives only, (7)-(9) can be rewritten as

$$
\frac{\partial}{\partial t} S_{A} \cdot V=S_{D} \cdot V
$$

where we have introduced the two relevant operators $S_{A}$ and $S_{D}$. For the electric and magnetic field, no spatial derivatives are present in the left hand sides of (7)-(8), $S_{A}$ is an operator which interpolates or averages the fields in space (according to the recipes of the previous subsection, also see Fig. 1). For the currents, no spatial derivatives occur in the constitutive equation (9), so they are not interpolated in space, and the current-current block of $S_{A}$ is a unit matrix. The remaining discretized space derivatives in Maxwell's equations and the current-current interactions are handled using the operator $S_{D}$. For Maxwell's curl equations, $S_{D}$ contains a combination of spatial derivatives and interpolations. For the current-current and electric-current interactions, it contains diagonal blocks containing $\omega_{s}$ and $\Omega$. Implicit time 
discretization of (24) gives:

$$
S_{A}\left(V_{t+1}-V_{t}\right) / \Delta_{t}=S_{D}\left(V_{t+1}+V_{t}\right) / 2
$$

This readily leads to the following relation between $V$ at time steps $n+1$ and $n$ :

$$
\left(S_{A}-S_{D} \Delta_{t} / 2\right) V_{t+1}=\left(S_{A}+S_{D} \Delta_{t} / 2\right) V_{t}
$$

We may interpret $\left(S_{A}-S_{D} \Delta_{t} / 2\right)$ as an operator which maps future fields back into the past by half a time step, and $\left(S_{A}+S_{D} \Delta_{t} / 2\right)$ as an operator which maps past fields into the future by half a time step. Equation (26) then demands that the past of the future equals the future of the past. We now have to carefully investigate if (26) can be solved for $V_{t+1}$ when $V_{t}$ is known. In order for this set of equations to be square, half of the electric field and magnetic field unknowns at the boundary of the studied domain must be determined by boundary conditions. This is the case when using periodic boundary conditions or PEC boundary conditions as in our examples.

\subsection{Stability}

Knowing that (26) can be solved for $V_{t+1}$ when $V_{t}$ is known of course allows to step through time, but we still have to proof that the obtained time-domain sequence is stable. In all our numerical simulations late time stability is indeed observed, but providing the most general proof turns out to be as yet elusive. In the sequel a proof is provided for 1D-propagation through a magnetized 1-specie plasma, when using periodic boundary conditions and this proof is then extended to $2 \mathrm{D}$ and $3 \mathrm{D}$ in section 8 .

Let the propagation direction be the x-direction and let us restrict ourselves to the mode where only $\mathcal{E}_{x}, \mathcal{E}_{z}, \mathcal{J}_{x}, \mathcal{J}_{z}$ and $\mathcal{B}_{y}$ are nonzero, i.e. the magnetic field is perpendicular to the propagation direction. Furthermore, we will use periodic boundary conditions in the $x$-direction, i.e. $f(x=0)=f(x=L)$ with $L$ the period and with $f$ each of the field components. 
We first define an interpolation matrix $M_{\text {int }}$ and a derivative matrix $M_{d e r}$ :

$$
\begin{aligned}
M_{i n t} & =\frac{1}{2}\left(\begin{array}{cccccc}
1 & 1 & 0 & 0 & 0 & \ldots \\
0 & 1 & 1 & 0 & 0 & \ldots \\
& & \vdots & & & \ddots \\
1 & 0 & 0 & \ldots & 0 & 1
\end{array}\right) \\
M_{\text {der }} & =\left(\begin{array}{ccccccc}
1 & -1 & 0 & 0 & 0 & \ldots \\
0 & 1 & -1 & 0 & 0 & \ldots \\
& & \vdots & & & \ddots \\
-1 & 0 & 0 & \ldots & 0 & 1
\end{array}\right)
\end{aligned}
$$

For $n$ discretization points these matrices are $n \times n$ matrices. They are circulant [16] because of the periodic boundary conditions. With these matrices the operators $S_{A}$ and $S_{D}$ become

$$
\begin{gathered}
S_{A}=\left(\begin{array}{ccccc}
1 & 0 & 0 & 0 & 0 \\
0 & M_{\text {int }} & 0 & 0 & 0 \\
0 & 0 & 1 & 0 & 0 \\
0 & 0 & 0 & 1 & 0 \\
0 & 0 & 0 & 0 & M_{\text {int }}
\end{array}\right) \\
S_{D}=\left(\begin{array}{ccccc}
0 & 0 & \omega_{p} & 0 & 0 \\
0 & 0 & 0 & M_{\text {int }} \omega_{p} & \frac{c}{\Delta} M_{\text {der }} \\
-\omega_{p} & 0 & 0 & \Omega & 0 \\
0 & -\omega_{p} & -\Omega & 0 & 0 \\
0 & \frac{c}{\Delta} M_{\text {der }} & 0 & 0 & 0
\end{array}\right)
\end{gathered}
$$

while $V_{t}=\left[\mathcal{E}_{x}, \mathcal{E}_{z}, \mathcal{J}_{x}, \mathcal{J}_{z}, \mathcal{B}_{y}\right]_{t}^{T}$. Remark that all "elements" in these matrices are themselves $n \times n$ matrices. Except for $M_{\text {int }}$ and $M_{\text {der }}$, they are all diagonal. Although $\omega_{p}$ and $\Omega$ are diagonal, not all diagonal elements need to be identical (i.e. the problem is not assumed to be uniform, the density and background magnetic field may be position-dependent). Mass lumping guarantees that $\omega_{p}$ and $\Omega$ are diagonal, but for the purposes of this proof it suffices that they are symmetric. We will now prove that all eigenvalues of the time-stepping operator $\left(S_{A}-S_{D} \Delta_{t} / 2\right)^{-1}\left(S_{A}+S_{D} \Delta_{t} / 2\right)$ are on the unit circle. Remark that

1. both $M_{\text {int }}$ and $M_{\text {der }}$ are circulant, so they are simultaneously diagonalisable using the Fourier basis [16]. We will call the $n \times n$ matrix that 
diagonalises them $F$. Furthermore, a general formula for their eigenvalues exists [16]. The $j$ th eigenvalue of a circulant $n \times n$ matrix with elements $m_{i, j}$ is

$$
\lambda_{j}=\sum_{k=1}^{n} m_{1, k} \exp \left(\frac{2 \pi i}{n} j(k-1)\right)
$$

where $j=0, \ldots, n-1$.

2. the eigenvalues of $M_{\text {int }}$ are $\lambda_{j}=\frac{1}{2}\left(1+\exp \left(\frac{2 \pi i}{n} j\right)\right)$ or $\exp \left(\frac{-j \pi i}{n}\right) \lambda_{j}=$ $\cos (\pi j / n)$ (Note that a zero eigenvalue exists only if $n$ is even. $M_{i n t}$ is invertible if $n$ is odd.)

3. the eigenvalues of $M_{d e r}$ are $\lambda_{j}=1-\exp \left(\frac{2 \pi i}{n} j\right)$ or $\exp \left(\frac{-j \pi i}{n}\right) \lambda_{j}=2 i \sin (\pi j / n)$.

Using $F, S_{A}$ and $S_{D}$ can be transformed into $\tilde{S}_{A}=T S_{A} T^{-1}$ and $\tilde{S}_{D}=$ $T S_{D} T^{-1}$, with

$$
T=\left(\begin{array}{ccccc}
F & 0 & 0 & 0 & 0 \\
0 & F & 0 & 0 & 0 \\
0 & 0 & F & 0 & 0 \\
0 & 0 & 0 & F & 0 \\
0 & 0 & 0 & 0 & F
\end{array}\right)
$$

$\tilde{S}_{A}$ is completely similar to $S_{A}$ with $M_{\text {int }}$ replaced by $D_{\text {int }}=F M_{\text {int }} F^{-1}$. After some calculations, using the fact that $c / \Delta$ in (30) is scalar and commutes with $F$ and with $M_{i n t}=F^{-1} D_{i n t}, \tilde{S}_{D}$ becomes

$$
\tilde{S}_{D}=\left(\begin{array}{ccccc}
0 & 0 & F \omega_{p} F^{-1} & 0 & 0 \\
0 & 0 & 0 & D_{\text {int }} F \omega_{p} F^{-1} & \frac{c}{\Delta} D_{\text {der }} \\
-F \omega_{p} F^{-1} & 0 & 0 & F \Omega F^{-1} & 0 \\
0 & -F \omega_{p} F^{-1} & -F \Omega F^{-1} & 0 & 0 \\
0 & \frac{c}{\Delta} D_{\text {der }} & 0 & 0 & 0
\end{array}\right)
$$

with $D_{\text {der }}=F M_{d e r} F^{-1}$. Let us now go back to (26). It is readily seen that (26) is equivalent to the very same equation in which $S_{A}$ and $S_{D}$ are replaced by $\tilde{S}_{A}$ and $\tilde{S}_{D}$. Multiplying both sides of this new equation with $\tilde{S}_{A}^{-1}$ gives:

$$
\left(1-\tilde{S}_{A}^{-1} \tilde{S}_{D} \Delta_{t} / 2\right) V_{t+1}=\left(1+\tilde{S}_{A}^{-1} \tilde{S}_{D}^{\prime} \Delta_{t} / 2\right) V_{t}
$$

Consider an eigenvector $v$ of $\tilde{S}_{A}^{-1} \tilde{S}_{D}$ with eigenvalue $\lambda$. This implies that

$$
\begin{aligned}
& \left(1-\tilde{S}_{A}^{-1} \tilde{S}_{D} \Delta_{t} / 2\right) v=\left(1-\lambda \Delta_{t} / 2\right) v \\
& \left(1+\tilde{S}_{A}^{-1} \tilde{S}_{D} \Delta_{t} / 2\right) v=\left(1+\lambda \Delta_{t} / 2\right) v
\end{aligned}
$$


hence $v$ is an eigenvector of $\left(1-\tilde{S}_{A}^{-1} \tilde{S}_{D} \Delta_{t} / 2\right)^{-1}\left(1+\tilde{S}_{A}^{-1} \tilde{S}_{D} \Delta_{t} / 2\right)$ with eigenvalue $\left(1-\lambda \Delta_{t} / 2\right) /\left(1+\lambda \Delta_{t} / 2\right)$. Provided that we can prove that $\lambda$ is purely imaginary, $\lambda=i \omega$, the eigenvalues of the fully discrete system become

$$
\frac{2-i \omega \Delta_{t}}{2+i \omega \Delta_{t}}
$$

and are on the unit circle for all $\Delta_{t}$. In the above reasoning we had to invoke the inverse of $\tilde{S}_{A}$ and hence, we must be sure that this inverse exists. If the number of discretizations $n$ is odd, this is always the case and for the further proof we will stick to this case (in the other case, stability also remains intact but a completely rigorous proof will not be provided here). What remains is the calculation of $\tilde{S}_{A}^{-1} \tilde{S}_{D}$. The result is

$$
\left(\begin{array}{ccccc}
0 & 0 & F \omega_{p} F^{-1} & 0 & 0 \\
0 & 0 & 0 & F \omega_{p} F^{-1} & \frac{c}{\Delta} D_{\text {int }}^{-1} D_{\text {der }} \\
-F \omega_{p} F^{-1} & 0 & 0 & F \Omega F^{-1} & 0 \\
0 & -F \omega_{p} F^{-1} & -F \Omega F^{-1} & 0 & 0 \\
0 & \frac{c}{\Delta} D_{\text {int }}^{-1} D_{\text {der }} & 0 & 0 & 0
\end{array}\right)
$$

Let us, e.g. take a closer look at $F \omega_{p} F^{-1}$. Not only are $F$ and $\omega_{p}$ symmetric, but the Fourier transform matrix $F$ has the special property that $F^{-1}=F^{*}$ (provided a $1 / \sqrt{N}$ factor is used in both the DFT and its inverse, with $N$ the number of points; if not so, $F^{-1}$ is proportional to $F^{*}$ and the proof remains valid). This implies that $F \omega_{p} F^{-1}=F \omega_{p} F^{*}$ and hence that $\left(F \omega_{p} F^{-1}\right)^{T}=$ $\left(F \omega_{p} F^{-1}\right)^{*}$, i.e. the real part of $F \omega_{p} F^{-1}$ is symmetric and its imaginary part is anti-symmetric. The same holds for $F \Omega F^{-1}$. The remaining quantity of interest in (38) is $\frac{c}{\Delta} D_{\text {der }} D_{\text {int }}^{-1}$. Using the eigenvalues of $M_{\text {int }}$ and $M_{d e r}$ as determined above, we have that

$$
\begin{aligned}
D_{\text {int }} & =\operatorname{diag}\left(1, \exp \left(\frac{2 \pi i}{n}(-1 / 2)\right), \exp \left(\frac{2 \pi i}{n}(-2 / 2)\right), \ldots, \exp \left(\frac{2 \pi i}{n}(-(n-1) / 2)\right)\right)^{-1} \\
& \cdot \operatorname{diag}(1, \cos (\pi 1 / n), \cos (\pi 2 / n), \ldots, \cos (\pi(n-1) / n)) \\
D_{d e r} & =\operatorname{diag}\left(1, \exp \left(\frac{2 \pi i}{n}(-1 / 2)\right), \exp \left(\frac{2 \pi i}{n}(-2 / 2)\right), \ldots, \exp \left(\frac{2 \pi i}{n}(-(n-1) / 2)\right)\right)^{-1} \\
& \cdot \operatorname{diag}(0,2 i \sin (\pi 1 / n), 2 i \sin (\pi 2 / n), \ldots, 2 i \sin (\pi(n-1) / n))
\end{aligned}
$$


and hence

$$
\begin{aligned}
D_{\text {der }} D_{\text {int }}^{-1} & =\operatorname{diag}(0,2 i \sin (\pi 1 / n), 2 i \sin (\pi 2 / n), \ldots, 2 i \sin (\pi(n-1) / n)) \\
& \cdot \operatorname{diag}(1, \cos (\pi 1 / n), \cos (\pi 2 / n), \ldots, \cos (\pi(n-1) / n))^{-1} \\
& =\operatorname{diag}\left(0, \frac{i}{2} \tan \left(\frac{\pi}{n}\right), \frac{i}{2} \tan \left(\frac{2 \pi}{n}\right), \ldots, \frac{i}{2} \tan \left(\frac{(n-1) \pi}{n}\right)\right)
\end{aligned}
$$

which is diagonal (symmetric) and purely imaginary. From all the above we conclude that the matrix (38) is skew-hermitian and has only purely imaginary eigenvalues $\lambda=i \omega$, as required.

Neither the amount of species nor the direction of the magnetic field were essential for this proof. Configurations with multiple species or a differently oriented $\overrightarrow{B_{0}}$ would merely give (much) larger matrices for $S_{A}$ and $S_{D}$.

For an extension of the proof to $2 \mathrm{D}$ and even $3 \mathrm{D}$ we refer the reader to the Appendix in Section 8.

\subsection{Choice of $\Delta$ and $\Delta_{t}$}

As a consequence of our way to deal with the space and time discretization, the discrete dispersion relation in vacuum is

$$
\left(\frac{\tan (k \Delta / 2)}{\Delta / 2}\right)^{2}=\frac{1}{c^{2}}\left(\frac{\tan \left(\omega \Delta_{t} / 2\right)}{\Delta_{t} / 2}\right)^{2}
$$

For sufficiently small $\Delta$ and $\Delta_{t}$ this dispersion relation reduces to the one for the continuous equations. However, remark that if $\Delta=c \Delta_{t}$

$$
\begin{aligned}
\left(\frac{\tan \left(k \Delta_{t} / 2\right)}{\Delta_{t} / 2}\right)^{2} / c^{2} & =\left(\frac{\tan \left(\omega \Delta_{t} / 2\right)}{\Delta_{t} / 2}\right)^{2} / c^{2} \\
\tan \left(k c \Delta_{t} / 2\right) & = \pm \tan \left(\omega \Delta_{t} / 2\right) \\
k c & = \pm \omega
\end{aligned}
$$

which is exactly equal to the continuous dispersion relation. The shortest resolvable wavelength is $\lambda=2 \Delta$ corresponding to $k=\pi / \Delta$. For this wavelength $\omega= \pm \pi / \Delta_{t}$. These values of $k$ and $\omega$ make the tangent infinite at both sides (42). Thus, the discrete dispersion relation $k=f(\omega)$ starts at $k= \pm \omega / c$ for small $k$ and $\omega$ and ends at $k=\pi / \Delta, \omega=\pi / \Delta_{t}$ for large values of $\omega$ (Fig. 2). In plasmas, too, high-frequency behaviour is vacuum-like and one can set $\Delta=c \Delta_{t}$ to make the discrete dispersion relation approximate the continuous dispersion relation even at high ill-resolved frequencies. 
While one of the advantages of an implicit approach is unconditional stability, i.e. the freedom to choose both $\Delta$ and $\Delta_{t}$ independently to fit frequencies and wavelengths under consideration, it may sometimes be useful to set $\Delta=c \Delta_{t}$ to get correct high-frequency behaviour all the way up to the Nyquist limit.

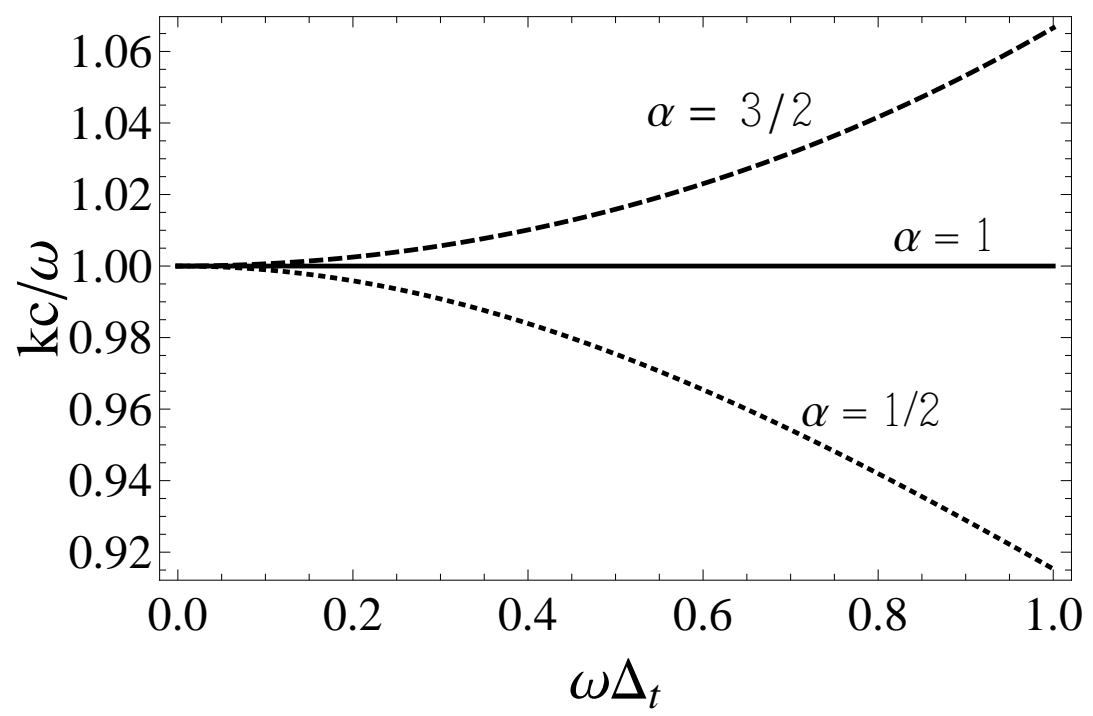

Figure 2: Vacuum discrete dispersion relation $(k \omega / c$ vs $\omega)$ for $\Delta=\alpha c \Delta_{t}$ with $\alpha=$ $1 / 2,1,3 / 2$. At low $k, \omega$ the behaviour is always approximately correct, but for $\alpha=1$ the behaviour is exact for all $\omega$.

\section{Optimizing the implicit step : a direct solution}

At first sight, a major drawback of the proposed method is the fact that we need the inverse of $\left(S_{A}-S_{D} \Delta_{t} / 2\right)$. However, let us partition $\left(S_{A}-S_{D} \Delta_{t} / 2\right)$ as follows

$$
\left(\begin{array}{cc}
E B & J E \\
E J & J J
\end{array}\right)
$$

Submatrix $E B$ contains all electric/magnetic interactions, $J E$ and $E J$ all electric/current interactions, and $J J$ the current-current interactions due to the background magnetic field. Thanks to mass lumping, the $J J$ part is purely local and hence block-diagonal implying that $(J J)^{-1}$ can easily be 
calculated explicitly. The inverse of $\left(S_{A}-S_{D} \Delta_{t} / 2\right)$ can then be expressed using the Schur complement $S=E B-(J E)(J J)^{-1}(E J)$ :

$$
\left(\begin{array}{cc}
E B & J E \\
E J & J J
\end{array}\right)^{-1}=\left(\begin{array}{cc}
S^{-1} & -S^{-1}(J E)(J J)^{-1} \\
-(J J)^{-1}(E J) S^{-1} & (J J)^{-1}+(J J)^{-1}(E J) S^{-1}(J E)(J J)^{-1}
\end{array}\right)
$$

Note that the dimension of $S$ (say $m \times m$ ) is already smaller than that of $S_{A}$ or $S_{D}$. The size of $S$ is independent of the number of particle species $N_{s}$, while the number of elements of $S_{A}$ and $S_{D}$ increases proportional to $N_{s}^{2}$. In practice, a sparse LU decomposition ${ }^{1}$ of the Schur complement is found to contain only $O(m)$ nonzero elements, i.e. $S x=y$ can be solved for $x$ in $O(m)$ if the LU decomposition is known. A direct solver based on formula (47) and an LU decomposition of the Schur complement runs orders of magnitude faster than a naive stabilized biconjugate gradient method to solve $\left(S_{A}-S_{D} \Delta_{t} / 2\right) x=y$. This is especially true for $c \Delta_{t} \gg \Delta$. For increasing $c \Delta_{t}$ the condition number of $\left(S_{A}-S_{D} \Delta_{t} / 2\right)$ increases and hence the amount of iterations needed for an iterative solution. However, this increase of $c \Delta_{t}$ barely influences the time needed for a direct solution using (47) and $S x=y$ at all. We will come back to this point in Section 5.2, providing some data to illustrate the above considerations.

\section{Numerical examples}

\subsection{A plasma wave beach in $1 D$ and $2 D$}

A first example of a non-uniform 1D problem in a 1-specie electron plasma is shown in Fig. 3. The density of the plasma increases as $x^{3}$ from $\omega_{p}=$ $\sqrt{\frac{n(x) q^{2}}{m \epsilon_{0}}}=0$ at the left (where $\left.n=0 \mathrm{~m}^{-3}\right)$ to $\omega_{p}=8 \omega$ at the right $(n=$ $3.4 \cdot 10^{18} \mathrm{~m}^{-3}$ ). The source frequency is $\omega=1.3 \cdot 10^{10} \mathrm{~s}^{-1}$ and the discretization length is $\Delta=1 \mathrm{~cm}$ and $\Delta_{t}=\Delta / c$ (at the Courant limit, about 14 samples per period). In an unmagnetized 1-specie plasma, waves only propagate if $\omega>\omega_{p}$, and indeed, in Fig. 3, we see that the waves propagate until they hit the position where $\omega=\omega_{p}$ and they stop propagating [8]. In this example, perfectly conducting (PEC) boundary conditions were used at $x=0$ and at

\footnotetext{
${ }^{1}$ Good sparse LU decomposition algorithms use permutation degrees of freedom to maximise the sparsity of the result while ensuring numerical stability, see e.g. [17]
} 
$x=200 \Delta$. This wave has only nonzero $E_{z}, J_{z}$ and $B_{y}$. A hard $E_{z}$ source is located at $x=50 \Delta$. The source is implemented as follows: first, the operator $\left(S_{A}+S_{D} \Delta_{t} / 2\right)$ acts on the fields $V$, i.e. $V_{n+1 / 2}=\left(S_{A}+S_{D} \Delta_{t} / 2\right) V_{n}$. Then, we set a single $E_{z}$ point in $V_{n+1 / 2}$ equal to our source value. Then we solve $\left(S_{A}-S_{D} \Delta_{t} / 2\right) V_{n+1}=V_{n+1 / 2}$.

A 2D 1-specie electron plasma wave beach is shown in Fig. 4. This example uses an $101 \times 101$ grid of discretization points and periodic boundary conditions (odd $\times$ odd guarantees the invertibility of $S_{A}$ and thus of $\left(S_{A}-\right.$ $\left.\frac{S_{D} \Delta_{t}}{2}\right)$ ). This wave has nonzero $E_{x}, E_{z}, J_{x}, J_{z}$ and $B_{y}$. The physical size is $1 \mathrm{~m} \times 1 \mathrm{~m}$. Again $\Delta_{t}=\Delta / c$. The source frequency is $\omega=1.3 \cdot 10^{10} \mathrm{~s}^{-1}$ and the density decreases like $\omega_{p}=\left(8.0 \cdot 10^{10} s^{-1}\right) \exp \left(-(d / 20)^{2}\right)$ where $d$ is the distance from the center (i.e. from the point at $(51 \Delta, 51 \Delta)$ ). This corresponds to a density of $n=2 \cdot 10^{18} \mathrm{~m}^{-3}$ at the center. At the edges, the density is at least three orders of magnitude lower. The source has a length of $20 \Delta$ and is at the left of the simulation region (due to the periodic boundary conditions, waves appear to enter the region from both sides). Again, we see that the waves encounter a cutoff condition and stop propagating. This example shows that it is in fact possible to use the proposed method in two or more dimensions.

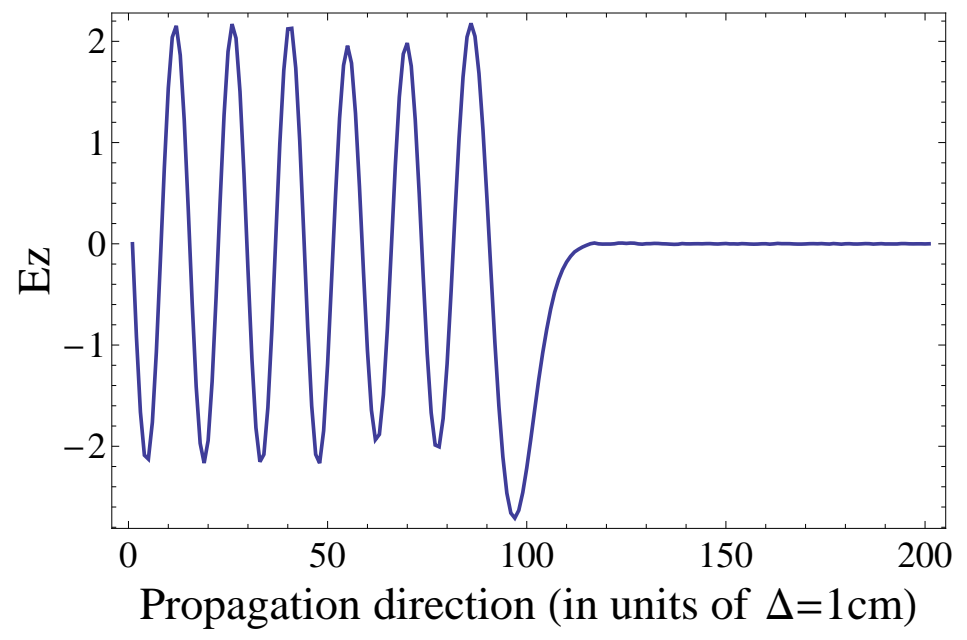

Figure 3: Waves in an unmagnetized 1-specie plasma with space-dependent density. As the density becomes too large, the wave can no longer propagate and is reflected, forming a standing wave to the left (low density) and zero electric fields to the right (high density). 

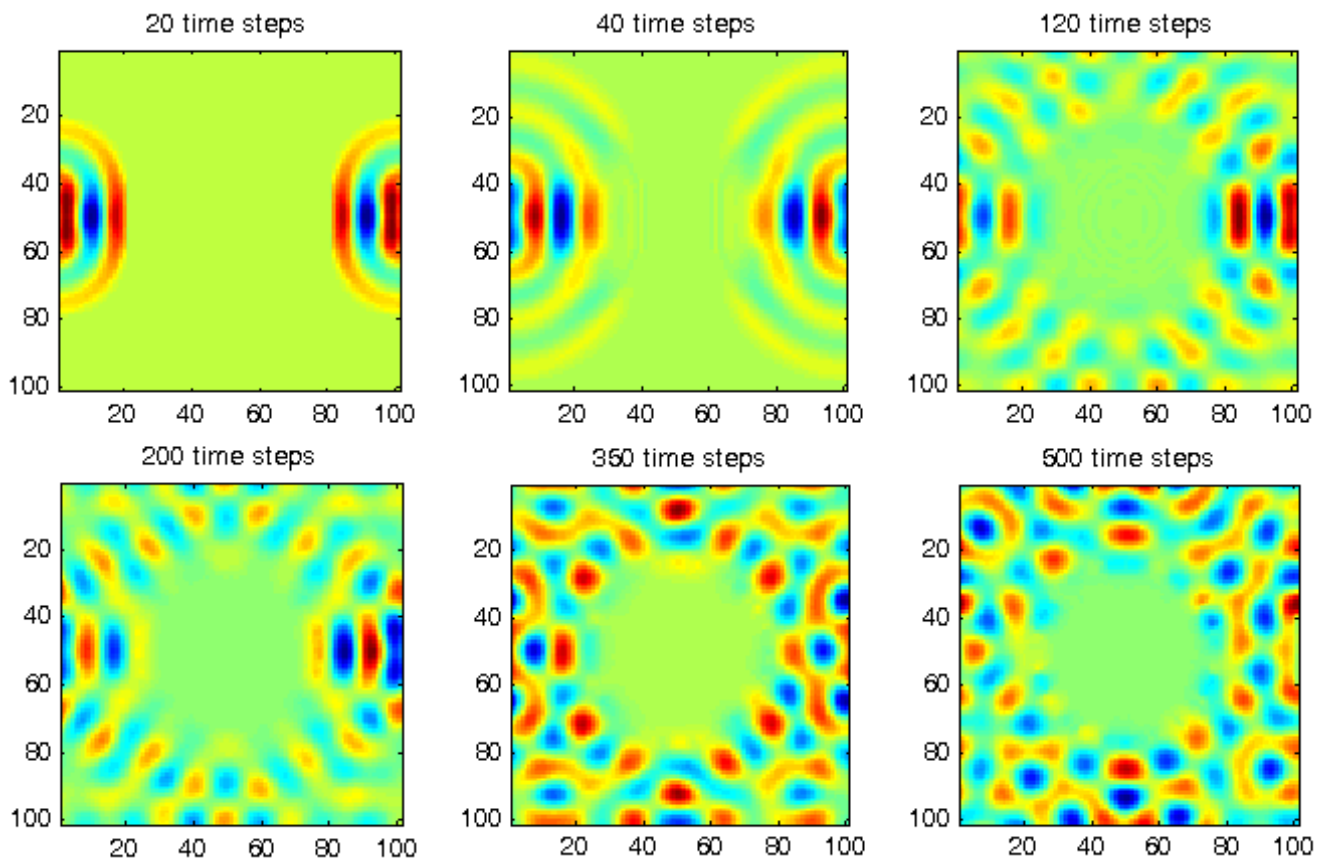

Figure 4: 2D configuration with waves propagating through a plasma whose density decreases radially (periodic boundary conditions).

\subsection{Mode conversion}

A third example involves mode conversion from the fast wave to the ioncyclotron wave, a phenomenon of interest for Ion-Cyclotron Resonance Heating (ICRH) $[9,10]$. This example is similar to the one used by Smithe [7]. In the simulations, we used PEC boundary conditions. As a matter of fact, we consider a simplified model of the behavior of a toroidal mode by replacing the angular dependence $\phi$ in the toroidal direction by the combination of a $\sin k_{y} y$ and $\cos k_{y} y$ dependence. In our example, $k_{y}=14.5 m^{-1}$. Furthermore, there is only an $x$-dependence and no $z$-dependence. This means that we have to use two sets of basis functions (modes): those of the form $r\left(x, x_{0}, \Delta_{x}\right) \sin \left(k_{y} y\right)$ and those of the form $r\left(x, x_{0}, \Delta_{x}\right) \cos \left(k_{y} y\right)$. The introduction of this modal dependence adds additional elements to the matrices (29) and (30), corresponding to the derivative in the $y$-direction. The plasma itself has the following parameters:

- $\left[B_{0 x}, B_{0 y}, B_{0 z}\right](x)=[0.15 B(x) \cos (\pi / 4), B(x), 0.15 B(x) \sin (\pi / 4)]$ where 
$B(x)$ decreases like $1 / x$ from $6.5 T$ at the far left (High Field Side or HFS) of the simulation domain to $5.5 T$ at the far right (Low Field Side or LFS). Further note that $B_{x}$ and $B_{z}$ cause coupling between the two modes

- Species: electrons $\left(n_{e}=2 \cdot 10^{20} m^{-1}\right)$, protons $\left(n_{p} / n_{e}=0.33\right)$, deuterons $\left(n_{d} / n_{e}=0.44\right),{ }^{3} \mathrm{He}-$ nuclei $\left(n_{\mathrm{He} 3} / n_{e}=0.115\right)$.

- Frequency $80.5 \cdot 10^{6} \mathrm{~Hz}$

- $\Delta_{x}=8 \cdot 10^{-4} m$

To get a better understanding of the physics of the problem, Fig. 5 shows a space-dependent dispersion relation. It was obtained by substituting fields with a $\exp \left(i k_{x} x\right) \exp \left(i k_{y} y\right) \exp (i \omega t)$ dependence into the continuous equations (7)-(9) in a uniform plasma, and then finding the values of $k_{x}$ for which a nontrivial solution exists (this involves solving a general quartic equation) with $k_{y}=14.5 m^{-1}$. At every position $x$, the plasma parameters are calculated and then $k_{x}(x)$ is obtained by assuming a uniform plasma with these parameters. As such, Fig. 5 is an approximation; it tells us which wavelength(s) to expect but does not give us the exact information (as would be the case for a uniform plasma). As the waves propagate to the right (LFS), shorter wavelengths (higher $k$ ) are excited.

Due to the presence of a background magnetic field that is not purely toroidal $\left(B_{x}, B_{z} \neq 0\right)$, the constitutive equation (9) enforces a relationship between all current components and as a consequence all cartesian components of the electric field, the magnetic field and currents are nonzero.

Despite there being only 200 discretization points in the $x$-direction, the time-step matrices are of size $7200 \times 7200$ : there are 6 vector fields to consider (electric, magnetic and 4 currents of the 4-species), every field is described by 2 modes and has 3 scalar components. Thus, there are $2 \times 3 \times 200$ unknowns per field, or $36 \times 200=7200$ unknowns in total ( 36 degrees of freedom per discretization point).

In Fig. 6, by way of example, one of the eigenmodes of the studied configuration is shown at a frequency of $80.5 \cdot 10^{6} \mathrm{~Hz}$. This result was obtained by calculating an eigenvector of the time-stepping operator $\left(S_{A}-S_{D} \Delta_{t} / 2\right)^{-1}\left(S_{A}+\right.$ $\left.S_{D} \Delta_{t} / 2\right)$ (at $c \Delta_{t}=\Delta_{x}$ ). Fig. 7 was obtained using time-stepping and a sinusoidal source of $80.5 \cdot 10^{6} \mathrm{~Hz}$ multiplied with a Heaviside step at $t=0$. 
In this figure, $c \Delta_{t}=100 \Delta_{x}$ which is far above the vacuum Courant condition (This choice of $\Delta_{t}$ corresponds to $46 \Delta_{t} \approx P=2 \pi / \omega$. Obeying the vacuum Courant condition at the same $\Delta_{r}$ would require $4600 \Delta_{t} \approx P$, i.e. vastly more time steps per period). Nonetheless the system remains stable as predicted. In both of the above figures we can clearly see the mode conversion from the fast to the ion-cyclotron wave, i.e. the appearance of short wavelength phenomena at the right.

For the above configuration we also investigated the efficiency of the algorithms for solving the sparse set of equations at every time step. Starting from a very crude discretization of 25 points in the $x$-direction, we gradually refined the discretization to 2500 points. We kept $\Delta_{x}$ constant and scaled the magnetic field variation such that $B(x)$ is always $6.5 \mathrm{~T}$ at the left and $5.5 \mathrm{~T}$ at the right, i.e. the magnetic field decrease is far steeper for a small amount of discretization points than for a large amount of discretization points. By doing this we ensure that $\lambda / \Delta_{x}$ remains in some sense constant. From Fig. 8, it is clear that the number of nonzero elements in the sparse LU-decomposition of the Schur complement $S$ (see (47)) is linear in the amount of discretization points, and thus provides an efficient solution method. In Fig. 9 the time needed for a direct solution (for 71 discretization points in the $x$-direction) is compared to the time needed for an iterative solution (using the iterative solvers and LU decomposition algorithms available in Matlab and ran on an Intel Core 2Quad CPU Q9650 3GHz processor) and this as a function of $c \Delta_{t} / \Delta_{x}$. It is clear that the time for the iterative solution increases as the time step increases beyond the vacuum Courant limit while the time needed for the direct solution stays constant.

\subsection{Dispersion relation in a uniform plasma}

In Fig. 10, the numerical dispersion relation for a uniform magnetized 4-specie plasma like that to the high-field side (far left) of Fig. 5 is shown as a function of the circular frequency $\omega$. We used periodic boundary conditions and calculated all eigenvectors and eigenvalues of the time-stepping operator. Thanks to uniformity and periodicity, the eigenvectors are pure complex exponentials, all with real $k_{x}$ (imaginary or complex $k_{x}$ cannot obey the periodic boundary conditions). For comparison, the real roots of the exact continuous dispersion relation in infinite space are shown too. 


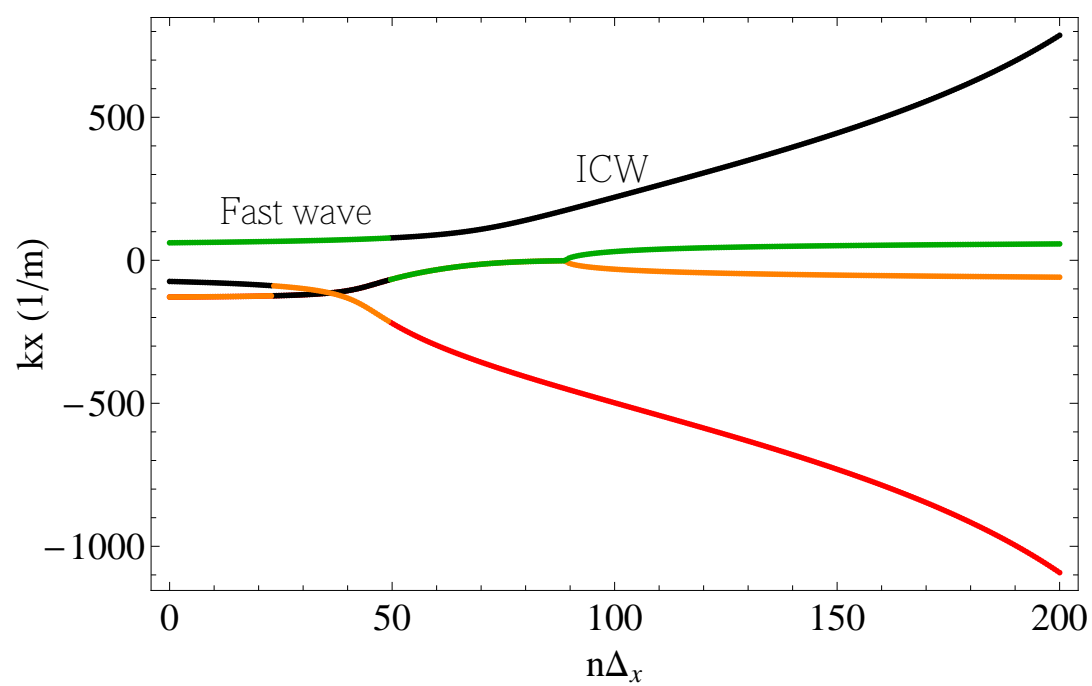

Figure 5: Space-dependent dispersion relation: $\Re\left(k_{x}\right)$ as a function of $n \Delta_{x}$ with mode conversion. Colours indicate the four solutions.
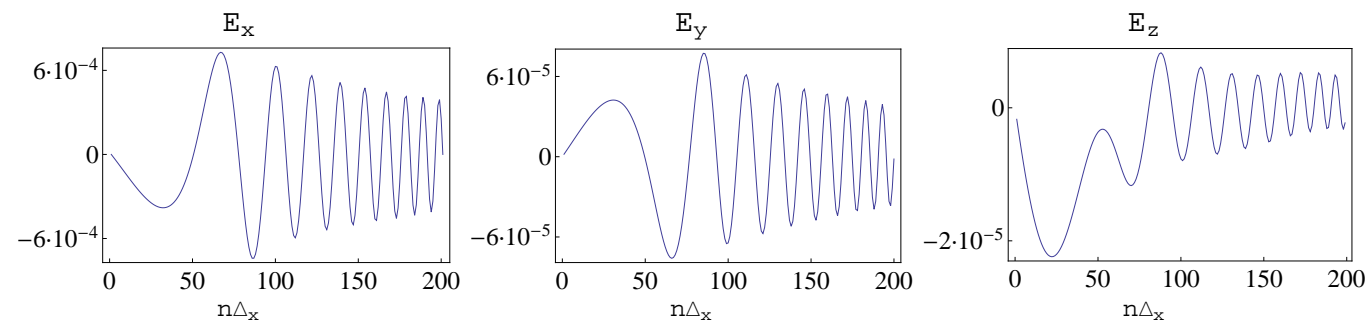

Figure 6: Real part of components of the electric field for an eigenmode of a plasma-filled cavity with mode conversion (at a frequency of $80.5 \cdot 10^{6} \mathrm{~Hz}$ ). 


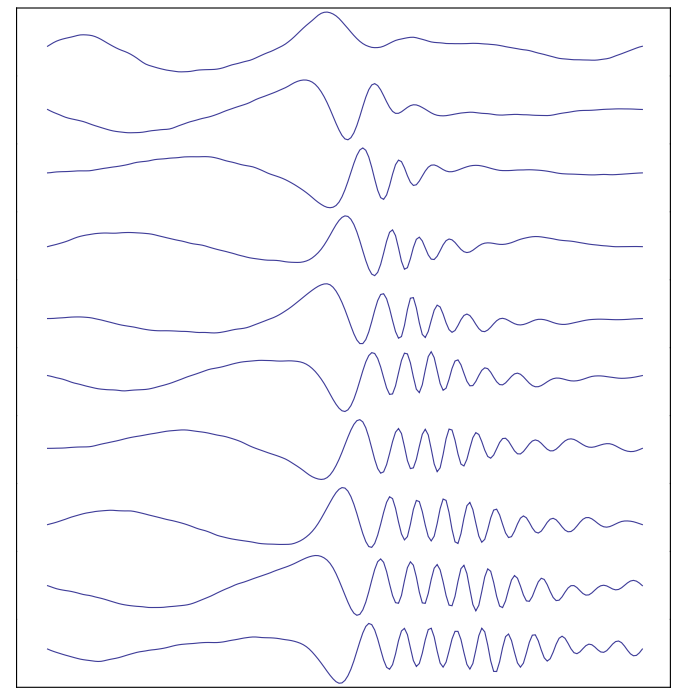

Figure 7: $E_{x}$ after 1000 (top),2000, ., 10000 (bottom) time steps, at $c \Delta_{t}=100 \Delta_{x}$. No attempt was made to filter out the desired mode at one particular frequency. This plot contains contributions of all resolvable frequencies in the Heaviside $(t) \sin (\omega t)$ source.
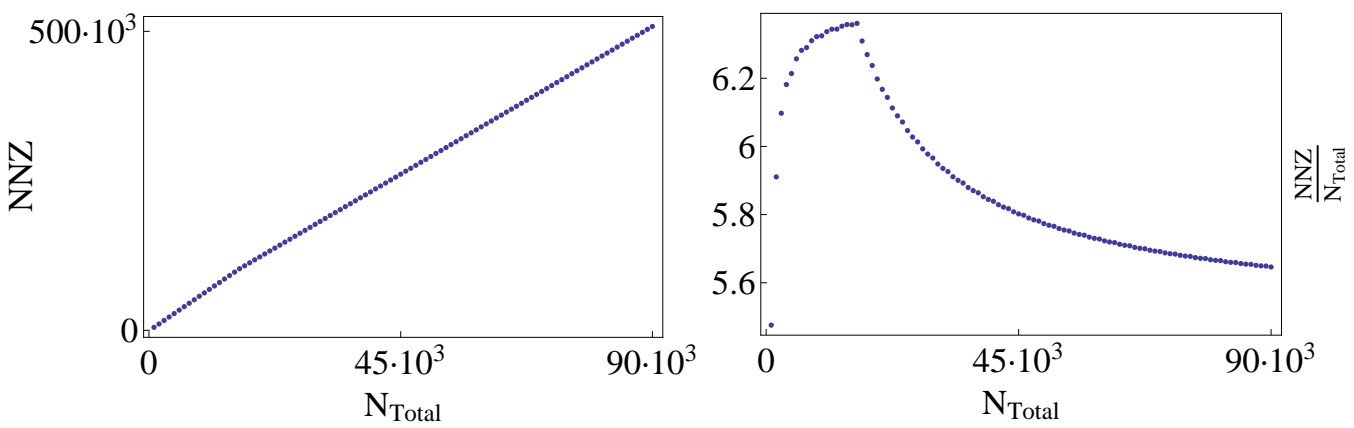

Figure 8: Absolute number of nonzero (NNZ) elements in the sparse LU decomposition of the Schur complement of $\left(S A-S D \Delta_{t} / 2\right)$ versus total amount of unknowns $N_{\text {total }}$ (left) and relative number (right). 


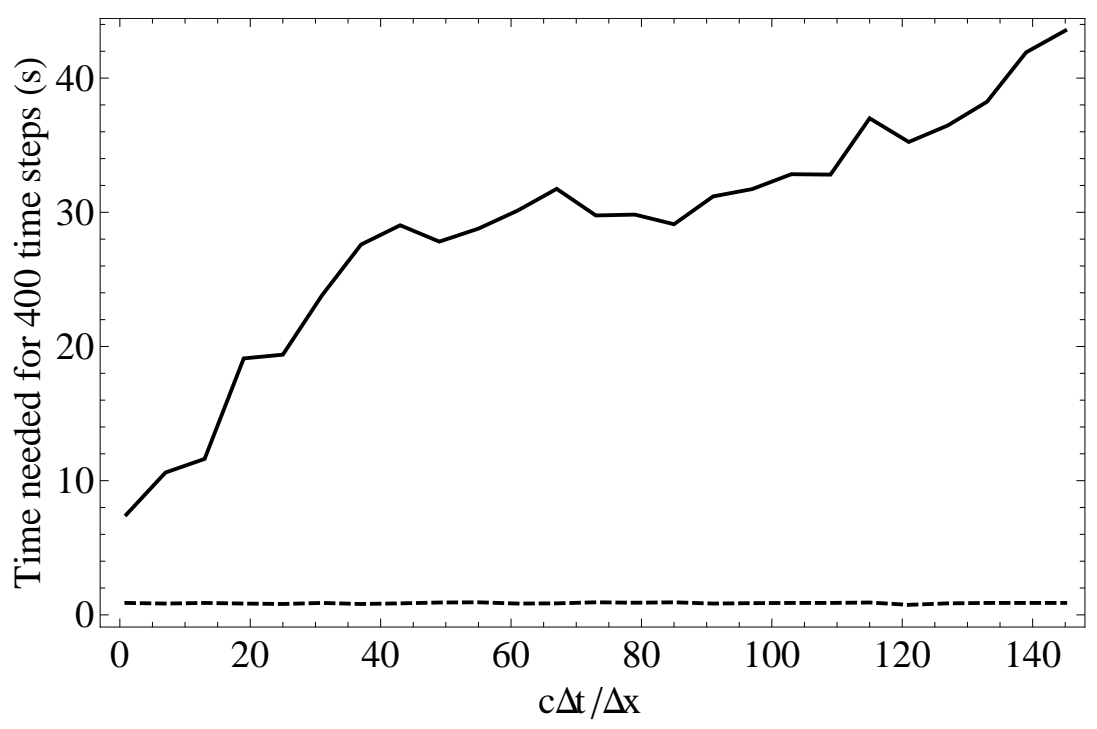

Figure 9: Time needed for a direct solution (dashed) vs. a naive stabilized biconjugate gradient (solid). (configuration of section 5.2, 71 discretization points). Initialisation time needed to calculate the LU decomposition of the Schur complement (direct case) or an incomplete LU preconditionner (iterative case) is not included on this graph. 


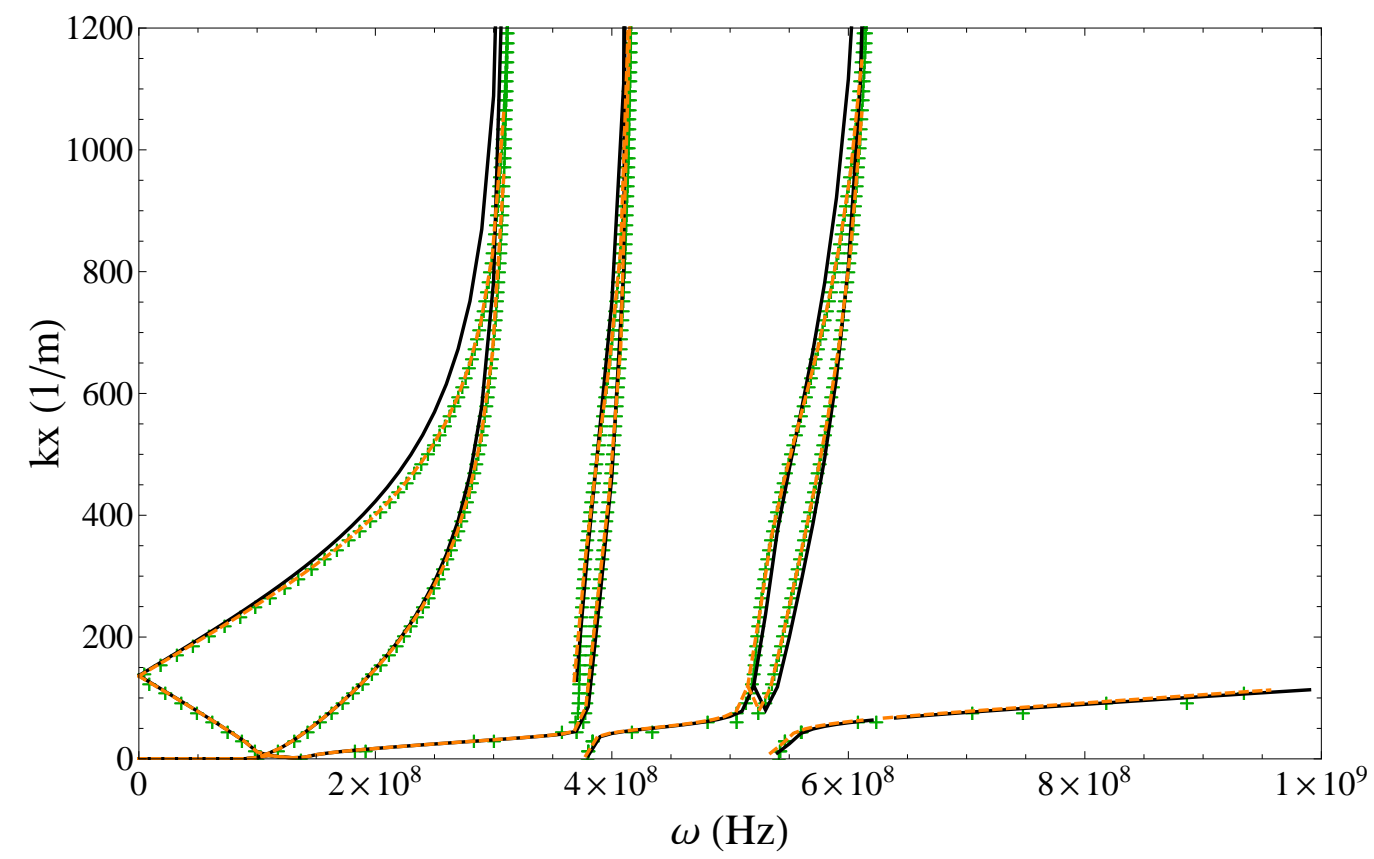

Figure 10: Green : Numerical value of $\left|k_{x}\right|$ versus $\omega$ obtained by calculating the eigenvectors of the system matrix and Fourier transforming them in space. The obtained $k_{x}$ is always real. Black: Analytically determined $\left|k_{x}\right|$ for propagating (real $k_{x}$ ) solutions of the exact dispersion relation. Orange: Analytically predicted discrete dispersion relation by replacing $\omega$ and $k_{x}$ by the relevant tangent functions in the continuous dispersion relation. 


\section{Conclusion}

In this paper we have presented an unconditionally stable time-domain discretization of Maxwell's equations complemented by a constitutive equation describing non-uniform magnetized cold plasma. The discrete dispersion relation of this discretization is guaranteed to be free of spurious modes and to approximate the continuous dispersion relation at well-resolved frequencies. We have provided a stability proof assuming 1D propagation and periodic boundary conditions and its extension to $2 \mathrm{D}$ and 3D. The method is implicit and requires the solution of a sparse set of equations every time step, but we have shown that this set of equations can, in practice, be solved very efficiently. We have demonstrated the effectiveness of this method using a number of examples, including a 1D and 2D plasma wave beach and mode conversion from the fast wave to the ion cyclotron wave. In classical FDTD, this last example would require very substantial oversampling in time due to the very short wavelengths compared to vacuum. Using our method, we can independently adapt the spatial and temporal discretizations to fit the phenomena under consideration, and we demonstrated this by running the mode conversion example at a time step $c \Delta_{t}=100 \Delta$ far above the vacuum Courant condition.

\section{References}

[1] R. M. Joseph, S. C. Hagness, A. Taflove, Direct time integration of Maxwell's equations in linear dispersive media with absorption for scattering and propagation of femtosecond electromagnetic pulses, Optics Letters (1991).

[2] M. W. Ammann, Non-Trivial Materials in EM-FDTD, Master's thesis, Swiss Federal Institute of Technology, Zurich, 2007.

[3] A. Taflove, S. Hagness, Computational Electrodynamics: The FiniteDifference Time-Domain Method,3rd ed., Artech House, 2005.

[4] F. Teixeira, Time-domain finite-difference and finite-element methods for maxwell equations in complex media, IEEE Transactions on Antennas and Propagation 56 (2008) 2150-2166. 
[5] S. H. Chang, A. Taflove, Finite-difference time-domain model of lasing action in a four-level two-electron atomic system, Optical Society of America (2004).

[6] X. Xi, R. Luo, J. Liu, J. Zhang, Y. Liu, Combined piecewise linear recursive convolution-bilinear transform implementation of the CFS-PML for unmagnetized plasma, IEEE Microw. Wireless Compon. Lett. 21 (2011).

[7] D. N. Smithe, Finite-difference time-domain simulation of fusion plasmas at radiofrequency time scales, Physics of Plasmas 14 (2007) 25372549 .

[8] T. Stix, Waves in Plasmas, American Institute of Physics, 1992.

[9] M. Mantsinen, M.-L. Mayoral, D. V. Eester, et al., Localized bulk electron heating with ICRF mode conversion in the JET tokamak, Nuclear Fusion (2004) 33-46.

[10] Y. Kazakov, I. Pavlenko, D. V. Eester, B. Weyssow, I. Girka, Enhanced ICRF (ion cyclotron range of frequencies) mode conversion efficiency in plasmas with two mode conversion layers, Plasma Physics and Controlled Fusion (2010).

[11] J. Hyman, M. Shashkov, Mimetic discretizations for Maxwell's equations, Journal of Computational Physics (1999).

[12] R. A. Chilton, H-, P- and T-refinement strategies for the finitedifference-time-domain (FDTD) method developed via finite-element (FE) principles, Ph.D. thesis, Ohio State University, 2008.

[13] A. Peterson, S. Ray, R. Mittra, Computational Methods for Electromagnetics, IEEE Press, 1998.

[14] S. C. Brenner, L. R. Scott, The mathematical theory of finite element methods, Springer, 2008.

[15] O. Sauter, Nonlocal analyses of electrostatic and electromagnetic waves in hot, magnetized, nonuniform, bounded plasmas, Ph.D. thesis, Ecole polytechnique federale de Lausanne, 1992. 
[16] R. M. Gray, Toeplitz and Circulant Matrices: A review, Stanford, CA:Stanford Univ, 2001.

[17] M. Bolhoffer, O. Schenk, Combinatorial aspects in sparse elimination methods, GAMM Mitteilungen (2004).

[18] C. F. V. Loan, The ubiquitous kronecker product, Journal of Computational and Applied Mathematics (2000) 85-100.

\section{Appendix: elaboration on the $2 \mathrm{D}$ and $3 \mathrm{D}$ case}

It is fairly straightforward to extend the proof given in Section 2.3 to $2 \mathrm{D}$ or even 3D. Consider an $n_{x} \times n_{z}$ problem like the one used in the 1D proof, but we no longer restrict the propagation to the $x$-direction: there may be propagation in the $z$-direction as well. Let $I_{x}$ be the $n_{x} \times n_{x}$ identity matrix, and $M_{i x}$ and $M_{d x} n_{x} \times n_{x}$ interpolation and derivative matrices as defined in (27) and (28) and define $I_{z}, M_{i z}$ and $M_{d z}$ in a similar way but now for the $z$-coordinate and replacing $n_{x}$ by $n_{z}$. The matrices (29) and (30) then become

$$
\begin{aligned}
& S_{A_{2 D}}=\left(\begin{array}{ccccc}
I_{x} \otimes M_{i z} & 0 & 0 & 0 & 0 \\
0 & M_{i x} \otimes I_{z} & 0 & 0 & 0 \\
0 & 0 & I_{x} \otimes I_{z} & 0 & 0 \\
0 & 0 & 0 & I_{x} \otimes I_{z} & 0 \\
0 & 0 & 0 & 0 & M_{i x} \otimes M_{i z}
\end{array}\right) \\
& S_{D_{2 D}}=\left(\begin{array}{ccccc}
0 & 0 & \omega_{p} I_{x} \otimes M_{i z} & 0 & \frac{c}{\Delta} I_{x} \otimes M_{d z} \\
0 & 0 & 0 & \omega_{p} M_{i x} \otimes I_{z} & \frac{c}{\Delta} M_{d x} \otimes I_{z} \\
0 & 0 & 0 & \Omega & 0 \\
-\omega_{p} & -\omega_{p} & -\Omega & 0 & 0 \\
0 & \frac{c}{\Delta} M_{d x} \otimes M_{i z} & 0 & 0 & 0
\end{array}\right)
\end{aligned}
$$

The pertinent derivative and interpolation matrices turn out to be Kronecker products of the previously defined $1 \mathrm{D}$ matrices. For the properties of the Kronecker product used here we refer to [18]. The plasma parameter matrices $\Omega$ and $\omega_{p}$ remain diagonal (and hence symmetric). Obviously $I_{x} \otimes I_{z}$ is the $\left(n_{x} n_{z}\right) \times\left(n_{x} n_{z}\right)$ identity matrix. Like in the $1 \mathrm{D}$ case, $S_{A_{2 D}}$ is invertible if both $n_{x}$ and $n_{z}$ are odd. To proceed as we did in the 1D proof, we must 
diagonalize matrices of the form $I_{x} \otimes M_{c z}(c=i, d), M_{c x} \otimes I_{z}$ and $M_{c x} \otimes M_{c z}$ where $M_{c x}$ and $M_{c z}$ are circulant. The identity matrix $I_{x}$ or $I_{z}$ is circulant too, thus it suffices to consider the most general case $M_{c x} \otimes M_{c z}$. This product is diagonalized by Kronecker products of DFT matrices. Indeed, if $F_{x}$ is the DFT matrix that diagonalizes $M_{c x}$ and $F_{z}$ the one that diagonalizes $M_{c z}$ then

$$
\begin{aligned}
\left(F_{x} \otimes F_{z}\right)\left(M_{c x} \otimes M_{c z}\right)\left(F_{x} \otimes F_{z}\right)^{-1} & =\left(F_{x} M_{c x} \otimes F_{z} M_{c z}\right)\left(F_{x}^{-1} \otimes F_{z}^{-1}\right) \\
& =\left(F_{x} M_{c x} F_{x}^{-1} \otimes F_{z} M_{c z} F_{z}^{-1}\right)
\end{aligned}
$$

which is a Kronecher product of diagonal matrices and hence itself diagonal. Knowing this, we may proceed just as in the $1 D$ case and verify that $S_{A_{2 D}}^{-1} S_{D_{2 D}}$ is anti-hermittian to prove stability. It is clear that the whole proof can be extended to 3D. 